\title{
Neuroimaging of the developing brain
}

\author{
John Darrell Van Horn • Kevin Archer Pelphrey
}

Published online: 28 February 2015

(C) Springer Science+Business Media New York 2015

Since its arrival as a critical tool for conducting the in vivo analysis of the human brain, interest has been paid to the neuroimaging of the developing brain (Davidson et al. 2003) and its use to explore clinical syndromes (Bookheimer et al. 1999). Indeed, modern magnetic resonance imaging (MRI) protocols and functional paradigms are an essential part of routine research and clinical neuroimaging in pediatric samples. Additionally, electrical recordings from the developing brain have also shown novel patterns in otherwise healthy subjects in contrast to those with developmental deficits (Jeste and Nelson 2009). The field of developmental neuroimaging and electrophysiological mapping continues to evolve, with greater emphasis on improved spatial resolution, more rapid data acquisition times, as well as a continued interest in mapping the development of functional aspects of the brain in prenatal as well as post-natal subjects.

The ability to noninvasively measure neuroanatomy, connectivity, and function in healthy young children, has already enhanced our understanding of brain and behavior relations (Casey et al. 2005; Holland et al. 2007; Nelson and McCleery 2008; Pelphrey et al. 2011). The application of these techniques to developmental research offers the opportunity to

\footnotetext{
J. D. Van Horn $(\bowtie)$

Institute for Neuroimaging and Informatics, University of Southern California, Keck School of Medicine of USC, 2001 North Soto Street, SSB1-102, Los Angeles, CA 90032, USA

e-mail: jvanhorn@usc.edu

K. A. Pelphrey

Center for Translational Developmental Neuroscience, Yale Child

Study Center, Yale University, 230 South Frontage Road, New

Haven, CT 06520, USA

e-mail: kevin.pelphrey@yale.edu
}

further explore these relationships and allows us to ask questions about where, when and how cognitive abilities develop in relation to changes in underlying brain systems. It is also possible to explore the contributions of maturation versus learning - as well as their interaction-in the development of these abilities through cross-sectional and longitudinal research involving training and intervention procedures. Current imaging methodologies, in conjunction with new and rapidly evolving techniques, hold the promise of even greater insights into developmental issues in the near future.

One major goal of these studies is to divine potential biomarkers for predicting altered neurodevelopmental outcomes and to inform/enhance clinical decision-making. Advanced MRI techniques such as diffusion tensor imaging, functional magnetic resonance imaging (fMRI), magnetic resonance perfusion, spectroscopy, volumetric imaging and arterial spin labeling collectively improve our understanding of normal brain development and pathophysiology of various adverse developmental processes. Coupled with evidence from electroencephalogram (EEG) recordings, such measures provide deep insights into functional and structural differences present in patients with autism spectrum disorder (ASD), attention deficit hyperactivity disorder (ADHD), issues of language comprehension, etc.

Studies of the brain in childhood are not simply equivalents of studies conducted on older subjects (Karmiloff-Smith 2010), however. The brains in question are smaller, with less imaging contrast, and possess other unique methodological considerations for imaging protocols (Luna et al. 2010). Though, neuroimaging studies also find ample evidence that adolescence is a period of continued neural development (Blakemore 2012), here, too, demographic, educational, and other factors are important elements for any investigation (Galvan et al. 2012). To be vexed by such problems belies, 
however, the enormous opportunity afforded by the examination of the brain as it grows, matures, and its functions refine and specialize (Luna et al. 2010).

Importantly, cross-sectional explorations using brain mapping methods provide evidence that the neural foundations of the behavioral signs of development involve not only integration of information across distributed brain networks but also basic functions in primary cortices (Wu et al. 2013). Longitudinal studies in ASD have shown abnormally enlarged brain volumes and increased rates of brain growth during early childhood, but only in a subset of ASD children (Marsh et al. 2008). Moreover, ASD symptoms have been hypothesized to result from altered brain connectivity (Delmonte et al. 2013). Several DTI studies reported microstructural differences indicative of developmental alterations in white matter organization, and potentially myelination, in ASD (Sundaram et al. 2008; Billeci et al. 2012; Kana et al. 2012). Altered structure within long-range white matter tracts linking socio-emotional processing areas has been strongly suggested (McPartland et al. 2011). This concept of developmental 'disconnectivity' might explain characteristic impairments in socio-emotional function, observed clinically in ASD. Similar work using MRI and electrophysiological approaches has suggested that the developing brain is at once a delicate and sensitive process, yet is surprisingly robust, adaptive, and plastic (Rossini and Dal Forno 2004). Neuroimaging has also made an impact in the study of language (Hoeft et al. 2011), social development (Sheridan et al. 2012), and even visual processing of action representation (Vander Wyk et al. 2012). Likewise, evoked potential EEG has shown alterations in face perception signals in children with ASD (Webb et al. 2011). Such studies are beginning to paint an interesting picture of the brain as it grows and the morphological, functional, and dysfunctional development which accompanies maturation.

In this special issue of Brain Imaging and Behavior, we have gathered a range of articles from top laboratories conducting research on brain development, patterns of altered structure and functional activity in patients with ASD, emerging methodological considerations for imaging in young children, electrophysiological effects, how to best interpret results, and how to access and interact with archives of primary neuroimaging data sets from the developing brain. The subjects of these articles were featured as part of the most recent New Horizons in Human Brain Imaging meeting held March 5-7, 2014 at the Turtle Bay Resort on the Island of Oahu, Hawaii USA. This 3-day international meeting of leading researchers from North America and Pacific Rim was comprised of key themes important to the next era of neuroimaging science, electrophysiology, and to address the measurement of prenatal, infant, and child brain development as well as common developmental disorders such as ASD and language abilities (for additional information, please visit http://www. pacificrimneuroimaging.net).
Horowitz-Kraus et al. report that correlation between percentile scores for the Woodcock-Johnson Passage Comprehension subtest and diffusion tensor imaging fractional anisotropy values in the right and left inferior longitudinal fasciculus and superior longitudinal fasciculus indicated positive associations in language-related regions of interest (ROIs), with greater distribution in the right hemisphere, which in turn showed strong connectivity in the fMRI data from the Sentence Picture Matching task. These results offer support for the participation of the right hemisphere in reading comprehension and may provide physiologic support for a distinction between different types of reading comprehension deficits vs. difficulties in technical reading.

In a study of EEG recording dynamics, Frohlich et al. explore the transients of waveform activity patterns observed in EEG time courses. They found that frequency variance was a promising marker of signal stability in younger children. EEG signal stability, as quantified by frequency variance, was found to increase with age in their sample of preschool age subjects. Future studies planned from this team are expected to relate this novel EEG biomarker with the development of executive function and cognitive flexibility in children, with the overarching goal of understanding electrical activity metastability in atypically developing children.

Sroka and coworkers comprising the Cincinnati MR Imaging of NeuroDevelopment (CMIND; http://research. cchmc.org/c-mind) consortium examined the functional imaging correlates associated with vocabulary ability and narrative comprehension in preschool children. Bilateral auditory cortex and superior temporal activation as well as left angular and supramarginal gyrus activation were observed during a passive listening-to-stories task. Boys exhibited greater activation than girls in the right anterior cingulate and right superior frontal gyrus. Finally, children with higher vocabulary scores showed increased grey matter leftlateralization and greater activation in bilateral thalamus, hippocampus, and left angular gyrus. Their examination illustrates a close association between left-hemisphere language regions and vocabulary scores in preschool-aged children using fMRI.

Ventola et al. investigated the mechanisms by which Pivotal Response Treatment (PRT) improves social communication in children with ASD. fMRI identified brain responses during a biological motion perception task conducted prior to and following 16 weeks of PRT treatment. Their findings support further investigation into the differential effects of PRT treatment strategies relative to specific neural targets. Identification of strategies for PRT which address neural vulnerability unique to each patient permits individualized interventions customized to the behavioral and neural characteristics in ASD children. 
McEvoy and colleagues quantified the potential effects of physiologic artifact on the estimation of EEG band power in a cohort of typically developing children. Such methods are needed in order to guide artifact rejection methods in quantitative EEG data analysis in developmental populations. The most significant differences in mean band power were found in the gamma band for electromyography (EMG) artifact and the theta band for ocular artifacts. Artifact detection strategies need to be sensitive to the oscillations of interest for a given analysis, with the most conservative approach being the removal of all EMG and ocular artifact from EEG data. Each of these variables appears differentially vulnerable to noise, and therefore, their interpretation depends on the methods used to identify and remove artifacts.

Douet and coworkers show here the genetic variations in the ERBB4 gene which are associated with increased susceptibility for schizophrenia and bipolar disorders. Structural imaging studies showed cortical abnormalities in adolescents and adults with schizophrenia and bipolar disorder in a very large cohort of children and adolescents (ages 3-20 years old; 462 girls and 509 boys). Subjects were genotyped for the ERBB4rs7598440 variants, had structural MRI, and cognitive evaluations. The ERBB4-TT-risk genotype children with no family history of mental health issues showed subtle cortical changes over age, primarily located in the left temporal lobe and superior parietal cortex. Conversely, the TT-risk genotype children with family history showed more pronounced age-related changes, mainly in the frontal lobes compared to the non-risk genotype children. Interaction effects of age, family history, and ERBB4 variations were also found on episodic memory and working memory, cognitive domains often impaired in schizophrenia and bipolar disorders. Such subclinical cortical alterations may pose as early indicators for increased risk of psychiatric disorders and provide additional understanding of the NRG1-ERBB4 pathway in brain development and childhood mental health.

Kundu et al. note that several methodological challenges affect the study of typical brain development based on resting state blood oxygenation level dependent (BOLD) in fMRI. These include increased head motion, in which younger subjects tend to be prone, age-dependence in cerebrospinal fluid (CSF) volume, vascular density, and CSF pulsation. They take the approach of a bottom-up revision of fMRI methodology based on acquisition of multi-echo fMRI and comprehensive utilization of the information in the TE-domain to enhance several aspects of fMRI analysis in the context of a developmental study. The analysis of multi-echo fMRI data eliminates a number of arbitrary processing steps such as bandpass filtering and spatial smoothing, while enabling procedures such as T2* mapping, BOLD contrast normalization and signal dropout recovery, precise anatomical-functional coregistration based on $\mathrm{T} 2 *$ measurements, automatic denoising through removing subject motion, scanner-related signal drifts and physiology, as well as statistical inference for seed-based connectivity. These benefits may be of significance and practical benefit for the study of typical and non-typical brain development.

Kaiser and colleagues evaluated the prevalence and type of unanticipated and potentially clinically significant imaging findings in a group of $N=114$ normal children enrolled in an ongoing MRI imaging study of normal brain development. Brain imaging measurements were classified using standardized scales and were then reported to participants and their primary healthcare provider according to a standard reporting pathway. Classification scales, reporting processes, and illustrated examples of findings are included and discussed. Unanticipated imaging findings were identified in over ten percent of children taking part in this study.

As a complement to the study by Kundu et al., Turner et al. also demonstrate that, not only does motion (among other confounds) exert an influence on the results of a BOLD variability analysis of task-related fMRI data- but, that the exact method used to deal with this influence has at least as large an effect as the motion itself. This sensitivity to relatively minor methodological changes is particularly concerning in younger subjects and mischaracterizing the relationship between BOLD variability and various individual differences.

Finally, Torgerson et al. discuss how the National Database for Autism Research (NDAR; http://ndar.nih.gov) provides open access to primary neuroimaging data, workflow methods, and high-performance computing will increase uniformity in data collection protocols, encourage greater reliability of published data, results replication, and broaden the range of researchers now able to perform larger studies than ever before. They illustrate the use of NDAR and the LONI Pipeline (http://pipeline.loni.usc.edu) workflow environment in performing several commonly performed neuroimaging processing steps and analyses useful for developmental neuroimaging investigators seeking to begin using this valuable combination of online data and computational resources.

In closing, the examination of the developing brain using advanced neuroimaging methods is rapidly providing new knowledge into the typical and atypical patterns of anatomical and connectomic change over time in addition to their functional alterations. Developmental syndromes, such as ASD, with putative morphological, connectomic, functional, and electrophysiological signatures in the brain can be readily examined, quantified, and compared with typically developing children. With this in mind, we invite the Brain Imaging and Behavior readership to enjoy this special collection of exceptional and timely articles on neuroimaging research into brain development. 


\section{References}

Billeci, L., Calderoni, S., Tosetti, M., Catani, M., \& Muratori, F. (2012). White matter connectivity in children with autism spectrum disorders: a tract-based spatial statistics study. BMC Neurology, 12, 148, pmcPmc3607981.

Blakemore S. J. (2012). Imaging brain development: the adolescent brain. NeuroImage, 61(2), 397-406.

Bookheimer S. Y., Dapretto M., \& Karmarkar U. (1999). Functional MRI in children with epilepsy. Developmental Neuroscience, 21(3-5), 191-199.

Casey B. J., Galvan A., \& Hare T. A. (2005). Changes in cerebral functional organization during cognitive development. Current Opinion in Neurobiology, 15(2), 239-244.

Davidson M. C., Thomas K. M., \& Casey B. J. (2003). Imaging the developing brain with fMRI. Mental Retardation and Developmental Disabilities Research Reviews, 9(3), 161-167.

Delmonte, S., Gallagher, L., O’Hanlon, E., McGrath, J., \& Balsters, J. H. (2013). Functional and structural connectivity of frontostriatal circuitry in Autism Spectrum Disorder. Frontiers in Human Neuroscience, 7, 430, pmcPmc3734372.

Galvan A., Van Leijenhorst L., \& McGlennen K. M. (2012). Considerations for imaging the adolescent brain. Developmental Cognitive Neuroscience, 2(3), 293-302.

Hoeft, F., McCandliss, B. D., Black, J. M., Gantman, A., Zakerani, N., Hulme, C., Lyytinen, H., Whitfield-Gabrieli, S., Glover, G. H., Reiss, A. L. \& Gabrieli, J. D. (2011). Neural systems predicting long-term outcome in dyslexia. Proceedings of the National Academy of Sciences of the United States of America, 108(1), 361-366, pmc3017159.

Holland S. K., Vannest J., Mecoli M., Jacola L. M., Tillema J. M., Karunanayaka P. R., Schmithorst V. J., Yuan W., Plante E., \& Byars A. W. (2007). Functional MRI of language lateralization during development in children. International Journal of Audiology, 46(9), 533-551.

Jeste S. S., \& Nelson 3rd C. A. (2009). Event related potentials in the understanding of autism spectrum disorders: an analytical review. Journal of Autism and Developmental Disorders, 39(3), 495-510.

Kana, R. K., Libero, L. E., Hu, C. P., Deshpande, H. D., \& Colburn, J. S. (2012). Functional brain networks and white matter underlying theory-of-mind in autism. Social Cognitive and Affective Neuroscience.
Karmiloff-Smith A. (2010). Neuroimaging of the developing brain: taking "developing" seriously. Human Brain Mapping, 31(6), 934941.

Luna, B., Velanova, K. \& Geier, C. F. (2010). Methodological approaches in developmental neuroimaging studies. Human Brain Mapping, 31(6), 863-871, pmcPmc2907666.

Marsh R., Gerber A. J., \& Peterson B. S. (2008). Neuroimaging studies of normal brain development and their relevance for understanding childhood neuropsychiatric disorders. Journal of the American Academy of Child and Adolescent Psychiatry, 47(11), 1233-1251.

McPartland, J. C., Coffman, M., \& Pelphrey, K. A. (2011). Recent advances in understanding the neural bases of autism spectrum disorder. Current Opinion in Pediatrics, 23(6), 628-632, pmc3413317.

Nelson, C. A., 3rd, \& McCleery, J. P. (2008). Use of event-related potentials in the study of typical and atypical development. Journal of the American Academy of Child and Adolescent Psychiatry, 47(11), 1252-1261, pmcPmc2791670.

Pelphrey, K. A., Shultz, S., Hudac, C. M., \& Vander Wyk, B. C. (2011). Research review: Constraining heterogeneity: the social brain and its development in autism spectrum disorder. Journal of Child Psychology and Psychiatry, 52(6), 631-644, pmc3096715.

Rossini P. M., \& Dal Forno G. (2004). Integrated technology for evaluation of brain function and neural plasticity. Physical Medicine and Rehabilitation Clinics of North America, 15(1), 263-306.

Sheridan, M. A., Sarsour, K., Jutte, D., D’Esposito, M., \& Boyce, W. T. (2012). The impact of social disparity on prefrontal function in childhood. PLOS ONE, 7(4), e35744, pmc3338535.

Sundaram S. K., Kumar A., Makki M. I., Behen M. E., Chugani H. T., \& Chugani D. C. (2008). Diffusion tensor imaging of frontal lobe in autism spectrum disorder. Cerebral Cortex, 18(11), 2659-2665.

Vander Wyk, B. C., Voos, A., \& Pelphrey, K. A. (2012). Action representation in the superior temporal sulcus in children and adults: an fMRI study. Developmental Cognitive Neuroscience, 2(4), 409416, pmc3431200.

Webb S. J., Jones E. J., Merkle K., Venema K., Greenson J., Murias M., \& Dawson G. (2011). Developmental change in the ERP responses to familiar faces in toddlers with autism spectrum disorders versus typical development. Child Development, 82(6), 1868-1886.

Wu, K., Taki, Y., Sato, K., Hashizume, H., Sassa, Y., Takeuchi, H., Thyreau, B., He, Y., Evans, A. C., Li, X., Kawashima, R., \& Fukuda, H. (2013). Topological organization of functional brain networks in healthy children: differences in relation to age, sex, and intelligence. PLoS ONE, 8(2), e55347, pmc3563524. 